\title{
Autonomy of lower-level perception from global processing in autism: Evidence from brain activation and functional connectivity
}

\author{
Yanni Liu ${ }^{\mathrm{a}, *}$, Vladimir L. Cherkassky ${ }^{\mathrm{a}}$, Nancy J. Minshew ${ }^{\mathrm{b}, \mathrm{c}}$, Marcel Adam Just ${ }^{\mathrm{a}}$ \\ a Center for Cognitive Brain Imaging, Department of Psychology, Carnegie Mellon University, Pittsburgh, PA 15213, United States \\ ${ }^{\mathrm{b}}$ Department of Psychiatry, University of Pittsburgh School of Medicine, Pittsburgh, PA, United States \\ ${ }^{\mathrm{c}}$ Department of Neurology, University of Pittsburgh School of Medicine, Pittsburgh, PA, United States
}

\section{A R T I C L E I N F O}

\section{Article history:}

Received 13 August 2010

Received in revised form 28 March 2011

Accepted 5 April 2011

Available online 13 April 2011

\section{Keywords:}

Autism

Local

Global

fMRI

Functional connectivity

\begin{abstract}
A B S T R A C T
Previous behavioral studies have shown that individuals with autism are less hindered by interference from global processing during the performance of lower-level perceptual tasks, such as finding embedded figures. The primary goal of this study was to examine the brain manifestation of such atypicality in high-functioning autism using fMRI. Fifteen participants with high-functioning autism and fifteen ageand IQ-matched typical controls were asked to perform a lower-level perceptual line-counting task in the presence of a distracting depiction of a 3-D object, in which participants counted whether there were more red or more green contours (In a contrasting 3-D task, participants judged whether the same 3-D stimulus objects (but without color in any contours) depicted a possible or impossible 3-D object). We hypothesized that individuals with autism would be less likely than controls to process the global 3-D information (and would hence show fewer neural signs of such interfering 3-D processing) during the lower-level linecounting task. The fMRI results revealed that in the line-counting task, the autism group did not show the increased medial frontal activity (relative to the possibility task), or the increased functional connectivity between the medial frontal region and posterior visual-spatial regions, demonstrated by the control group. Both findings are indices of lesser effort and difficulty in the line-counting task for the autism group than for the control group, attributed to less interference from the 3-D processing in the autism group. In addition, in the line-counting task, the control group showed a positive correlation between a measure of spatial ability (Vandenberg scores) and activation in the medial frontal region, suggesting that more spatially able control participants did more suppression of the irrelevant 3-D background information in order to focus on the line-counting task. The findings collectively indicate that the global 3-D structure of the figure had a smaller effect, if any, on local processing in the group with autism compared to the control group. The results from this study provide the first direct neural evidence of reduced global-to-local interference in autism.
\end{abstract}

(C) 2011 Elsevier Ltd. All rights reserved.

\section{Introduction}

Autism is a neurodevelopmental disorder characterized by core deficits in communication and social cognition, in the presence of repetitive behavior and limited imagination (APA, 1994). Individuals with autism have also shown some atypical perceptual processing, often demonstrating superior performance in tasks requiring the processing of fine stimulus features rather than stimulus configurations. For example, participants with autism can better detect hidden figures than normal controls (Jolliffe \& BaronCohen, 1997) and show enhanced detection of a local target in a visual search task (O'Riordan, Plaisted, Driver, \& Baron-Cohen, 2001 ; Plaisted, O’Riordan, \& Baron-Cohen, 1998). In face processing,

\footnotetext{
* Corresponding author. Tel.: +1 412268 3784; fax: +1 4122682804

E-mail address: yanniliu.cmu@gmail.com (Y. Liu).
}

they focus on individual face features rather than the configuration (Hubl et al., 2003; Joseph \& Tanaka, 2003). Meanwhile they do not benefit from the segmentation of visual stimuli in the block-design task (Shah \& Frith, 1993) and use a gestalt heuristic significantly less often than controls do (Brosnan, Scott, Fox, \& Pye, 2004).

Three related accounts have been advanced to interpret these abnormalities in perception in autism. One is the weak central coherence (WCC) account, which originally proposed a deficit in autism in processing the global aspects of information, such that this weak "central coherence" led to a bias for processing featural and local information (Frith, 1989). Recently the hypothesis has moved towards an emphasis on superiority in local processing rather than on deficit in global processing (Happé \& Frith, 2006). However, the latest versions of the WCC account reemphasized the notion of reduced global integration of information (Happé \& Booth, 2008). Second, Plaisted $(2000,2001)$ hypothesized that superior perceptual discrimination in autism underlies the pattern 
of the hyper-function in some perceptual tasks. Third, the enhanced perceptual functioning (EPF) theory (Mottron, Dawson, Soulières, Hubert, \& Burack, 2006), proposed that the default setting of autistic perception is more locally oriented than that of controls. While Plaisted's superior perceptual discrimination view did not discuss hierarchical processing, both the WCC and EPF accounts deal with the atypical relationship between local and global processing. The EPF theory and recent WCC accounts share the idea that individuals with autism implicitly place a greater focus on local processing than on global processing in open-ended tasks. While the EPF theory suggests that individuals with autism are capable of global processing when it is called for, the WCC theory is indecisive of the impairment of the global processing.

One way that a bias towards local processing can manifest itself is in a task that requires a local focus, but nevertheless contains global information that tends to be processed automatically. In this circumstance, the local bias and non-mandatory global processing can result in an absence of interference from the global level of processing. That is, people with autism may be less hindered than controls by interference from the processing of task-irrelevant global information. For example, individuals with autism are better at copying impossible figures than are controls (Mottron, Belleville, \& Ménard, 1999), they complete the block design tasks faster and with fewer mistakes than controls when presented with unsegmented geometric designs (Shah \& Frith, 1993), and they are less influenced by the number of distractors in visual search tasks (O'Riordan et al., 2001). However, their performance when working with global-level stimuli is more affected by incongruent stimuli at the local level (Behrmann et al., 2006; Rinehart, Bradshaw, Moss, Brereton, \& Tonge, 2000).

While most evidence concerning a local bias comes from behavioral measures, there are a few neuroimaging studies that have explored atypical perception in hierarchical processing in autism, mainly using the embedded figures task (EFT), in which participants are asked to mentally decompose a complex figure into local parts in order to decide whether one of the parts matches the target figure. Ring et al. (1999) found greater activation of ventral occipitotemporal regions in autism and greater prefrontal activity in controls and suggested that the two groups may adopt different cognitive strategies to perform the EFT, particularly that the autism group depends, to an abnormally large extent, on visual systems for object feature analysis. However, the data are not easy to interpret due to the small sample size (with six individuals with autism) and a not-well-matched control group. Manjaly et al. (2007) contrasted brain activation in response to the EFT with a closely matched visuospatial control task in adolescent population, and found that task-specific activation was left-lateralized in parietal and premotor areas for controls, but was found in right primary visual cortex and bilateral extrastriate areas in autism. They concluded that enhanced local processing, rather than impaired processing of global context, is characteristic of performance in the EFT by patients with autism. Lee et al. (2007) found that children with autism show generally reduced cortical involvement, but the same level of behavioral performance as controls in the EFT, which does not clearly implicate whether it is reduced global or enhanced local processing that underlies perceptual processing in autism. Damarla et al. (2010) have shown greater activation of visuospatial areas in autism and greater activation of left dorsolateral prefrontal and inferior parietal areas in controls in an EFT. In addition, they observed reduced frontal-posterior functional connectivity in autism, suggesting that the integration of higher-order executive/working memory brain regions with visuospatial regions might be impaired in individuals with autism in EFT.

In order to specify the activation related to visual perception of complex spatial information and disembedding processing in the
Table 1

Demographic information for autism and control groups.

\begin{tabular}{llllll}
\hline & & Autism & Control & $t$ Value & Pr \\
\hline Age (years) & Mean \pm SD & $25.2 \pm 7.6$ & $26.3 \pm 8.2$ & 0.38 & ns \\
VIQ & Mean \pm SD & $104.5 \pm 13.1$ & $110.0 \pm 3.8$ & 1.57 & ns \\
PIQ & Mean \pm SD & $116.1 \pm 10.1$ & $111.6 \pm 6.8$ & 1.45 & ns \\
FSIQ & Mean \pm SD & $111.2 \pm 10.0$ & $112.3 \pm 4.5$ & 0.38 & ns \\
Vandenberg & Mean \pm SD & $23.8 \pm 9.9$ & $22.3 \pm 7.1$ & 0.49 & ns \\
Handedness & Right:left & $15: 0$ & $15: 0$ & & \\
Gender & Male:female & $14: 1$ & $15: 0$ & & \\
\hline
\end{tabular}

EFT, researchers (Lee et al., 2007; Manjaly et al., 2003, 2007) often use a visuospatial control task with very similar stimuli controlling for the motor, cognitive, and perceptual process involved in the EFT, except for the local search requirement. However, this type of design does not distinguish between global interference impacting the disembedding processing and global processing of the complex spatial layout itself. The present study aimed at providing direct neuroimaging evidence regarding reduced global-to-local interference in autism.

In our experiment, we presented depictions of the same objects (with some minor color differences) in two different tasks. The stimuli depicted possible or impossible 3-D objects, where several contours were colored in the line-counting task. The line-counting task (count task) required participants to simply count the colored lines, a form of more local visual processing. We expected that while performing the count task, some participants would nevertheless automatically process the 3-D structure of the object. The possibility task, in which the participants were asked to judge whether the drawing depicted a possible or impossible 3-D object, was intended to focus attention on the 3-D structure of the object, which is inherently a more global type of processing. Our goal was to study how the automatic 3-D processing during the line-counting task might interact with the task-required local processing for the two groups of participants. The possibility task served as a comparison condition that explicitly required all participants to perform the 3-D (more global) processing.

We used functional MRI to explore the brain manifestation of the reduced global-to-local interference in autism perception by contrasting the brain activation in the count task with the possibility task. In typical controls, the mandatory/automatic global processing of the background information in the count task may interfere with the concurrent local processing, causing additional cognitive resources to be recruited to deal with the interference. Thus, greater activation in the executive brain regions and increased functional connectivity between the executive regions and the visuospatial regions are expected to be found in controls in the count task. The relative autonomy of lower-level processing and a processing bias for featural and local information in autism should result in less interference from task-irrelevant global information affecting the count task compared to controls.

\section{Methods}

\subsection{Participants}

The participants were 15 adults with high-functioning autism (14 males and 1 female) and 15 typical control participants (15 males). They were matched for age, gender, and Full Scale, Verbal, and Performance IQ, as determined by the Wechsler Adult Intelligence Scale Revised (WAIS-R). The diagnosis of autism was established using two structured research diagnostic instruments: the Autism Diagnostic Interview-Revised (ADI-R; Lord, Rutter, \& Le Couteur, 1994) and the Autism Diagnostic Observation Schedule-Generic (ADOS-G; Lord et al., 2000), supplemented with expert clinical opinion according to accepted criteria of high-functioning autism (Minshew, 1996) (Table 1).

Potential participants with autism were excluded if they had an identifiable cause for their autism, such as fragile-X syndrome, tuberous sclerosis, or fetal cytomegalovirus infection. All potential participants (autism or control) were excluded if there was evidence of birth asphyxia, head injury, or a seizure 
Line-Counting (More Red More Green)

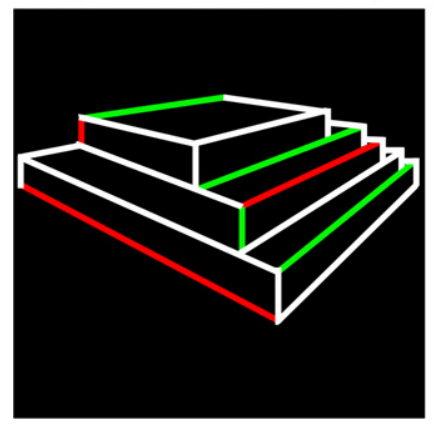

Possibility Judgement (Possible Impossible)

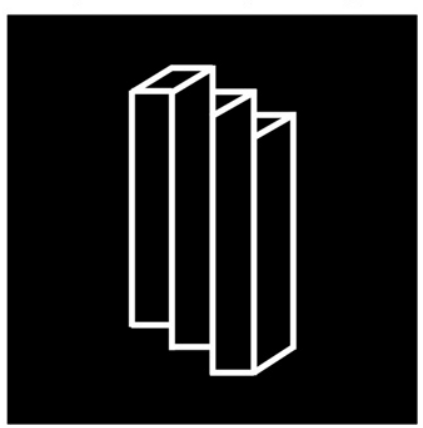

Fig. 1. Examples of experimental stimuli. Left: a possible 3-D figure with colored contours in the line-counting task; right: an impossible 3-D figure in the possibility judgment task.

disorder. Exclusionary criteria were based on neurologic history and examination, and chromosomal analysis, or metabolic testing, if indicated.

Potential control participants were screened by questionnaire, telephone, face-to-face interview, and observation during screening psychometric tests. Exclusionary criteria, evaluated through these procedures, included current or past psychiatric and neurologic disorders, birth injury, developmental delay, school problems, acquired brain injury, learning disabilities, substance abuse, and medical disorders with implications for the central nervous system or those requiring regular medication. Potential control participants were also screened to exclude those with a family history (in parents, siblings, and offspring) of autism, developmental cognitive disorders, affective disorders, anxiety disorders, schizophrenia, obsessive compulsive disorder, substance abuse, or other neurologic or psychiatric disorders thought to have a genetic component. Handedness was determined with the Lateral Dominance Examination from the Halstead-Reitan Neuropsychological Test Battery (Reitan, 1985). All participants were right-handed.

\subsection{Experimental paradigm}

There were two experimental tasks: (1) line-counting task, in which red and green contours were drawn on a possible or impossible 3-D object, and participants were asked to count and decide whether there were more red or green lines; and (2) possibility judgment task, in which participants were presented with a complex line drawing to be judged as a possible or impossible 3-D object. Sample stimuli are shown in Fig. 1. Each task included two blocks of stimuli, with ten trials in each block. Tasks were presented in one of two sequential orders: ABBA or BAAB. Participants were randomly assigned to one of the sequences. A fixation asterisk $\left({ }^{*}\right)$ was presented in the middle of the screen for five $24 \mathrm{~s}$ periods, with one before the first block and the other four following each task block, respectively.

There were equal numbers of possible and impossible figures in both the count and possibility tasks. Preliminary analyses indicated that the possibility variable yielded no group differences or interesting results, thus possibility was not further treated as an experimental factor in this paper. In the count task, half of the trials had more red lines and the other half had more green lines. In most trials (17 out of 20), three lines of one color and four lines of the other color were presented; in the other trials, four lines of one color and five lines of the other color were presented.

For each trial, the stimulus was presented for $8 \mathrm{~s}$, followed by a $7 \mathrm{~s}$ rest (signaled by an asterisk in the middle of the screen). Participants were asked to respond during the $8 \mathrm{~s}$ presentation. In the count task, more red and more green judgments were matched to left and right index finger responses, respectively. In the possibility task, possible and impossible judgments were indicated by left and right index finger responses, respectively.

Prior to entering the scanner, the participants completed a practice session consisting of 20 items with feedback and 12 items without feedback (equal number of items for each task). The figures used in the practice session were not used in the actual experiment.

\subsection{Vandenberg test}

Visual-spatial ability was assessed through the Vandenberg Test of Mental Rotation (Vandenberg \& Kuse, 1978; maximum score =40), which uses figures originally designed by Shepard and Metzler (1971). The task involves correctly matching a target 3-D figure to correct depictions of the same figure in a different orientation. The testing was performed outside the scanner before the participants completed the practice session, allowing $3 \mathrm{~min}$ to complete each of the two sections of the test.

\section{4. fMRI procedures}

The data were collected using a 3-T Siemens Allegra scanner (Siemens, Erlangen, Germany) at the Brain Imaging Research Center of Carnegie Mellon University and the University of Pittsburgh. The stimuli were rear-projected onto a translucent plastic screen, and participants viewed the screen through a mirror attached to the head coil. The study was performed with a gradient echo, echo-planar pulse sequence with $\mathrm{TR}=1000 \mathrm{~ms}, \mathrm{TE}=30 \mathrm{~ms}$, and a $60^{\circ}$ flip angle. Seventeen adjacent oblique-axial slices were acquired in an interleaved sequence; each slice was $5-\mathrm{mm}$ thick with a gap of $1 \mathrm{~mm}$ between slices. The voxel size was $3.125 \mathrm{~mm} \times 3.125 \mathrm{~mm} \times 5 \mathrm{~mm}$.

\section{5. fMRI analyses}

\subsubsection{Distribution of activation}

To compare the participating groups in terms of the distribution of activation, the data were analyzed using SPM2. Images were corrected for slice acquisition timing, motion-corrected, normalized to the Montreal Neurological Institute (MNI) template, resampled to $2 \mathrm{~mm} \times 2 \mathrm{~mm} \times 2 \mathrm{~mm}$ voxels, and smoothed with an 8-mm Gaussian kernel to decrease spatial noise. Statistical analysis was performed on individual and group data using the general linear model and Gaussian random field theory as implemented in SPM2 (Friston et al., 1995). Group analyses were performed using a random-effects model. An uncorrected height threshold of $p<0.001$ and an extent threshold of six $8 \mathrm{~mm}^{3}$ voxels were used.

\subsubsection{Functional connectivity}

The functional connectivity was computed (separately for each participant) as a correlation between the average time course of signal intensity of all the activated voxels (thresholded at $t=3.0$ ) in each member of a pair of regions of interest (ROIs). Twenty-one functional ROIs were defined to encompass the main clusters of cortical activation in the group activation map for each group in both tasks vs. fixation. Labels for these 21 ROIs (the medial frontal cortex and 10 bilateral ROIs, namely dorsolateral prefrontal, inferior frontal, middle frontal, fusiform, insula, inferior parietal, postcentral, precuneus, inferior occipital, and middle occipital) were assigned with reference to the parcellation of the Montreal Neurological Institute (MNI) single-subject, T1-weighted dataset reported by Tzourio-Mazoyer et al. (2002). For each of these ROIs, a sphere was defined (with a radius ranging from $8 \mathrm{~mm}$ to $14 \mathrm{~mm}$ ) that best captured the cluster of activation in the map for each group. Each ROI used in the analysis was composed of the union of the two spheres defined for the two groups. The functional connectivity was computed in the following steps: first, the average time courses were extracted from each participant over the activated voxels within each ROI during task performance, excluding the fixation conditions. This extraction originated from the normalized and smoothed images, which were high-pass filtered and had the linear trend removed. Second, the correlations were computed on the extracted time courses among ROI pairs, separately for the count and possibility tasks. Third, Fisher's $r$-to- $z$ transformation was applied to the correlation coefficients for each participant. Fourth, for each participant, the mean functional connectivity of various sets of ROI pairs (such as all frontal-posterior pairs) was computed. Fifth, these mean functional connectivities were statistically compared. The term that has been used throughout the fMRI field for this measure for many years is functional connectivity, but what would have been more accurate is synchronization of activation. We will use the two terms interchangeably.

\subsubsection{Percent signal change}

The percent signal change was computed for each participant as the percent increase in signal over all voxels in a functional ROI during each of the tasks relative to the signal during fixation. The signal extracted from the functional ROI originated from the normalized and smoothed images, which were high-pass filtered and had the linear trend removed.

\section{Results}

\subsection{Overview}

Despite comparable behavioral performance in the two groups, the predicted interactions between group and task occurred for both the amount of activation (in superior medial frontal areas) and the level of functional connectivity (synchronization) between activated areas. For both of these dependent measures, the autism group, unlike the control group, did not show an increase in the local (line-counting) task, indicating this group's relatively lower level of difficulty and computational load in this condition. 
(a) Differences of Activation Between the Two Tasks

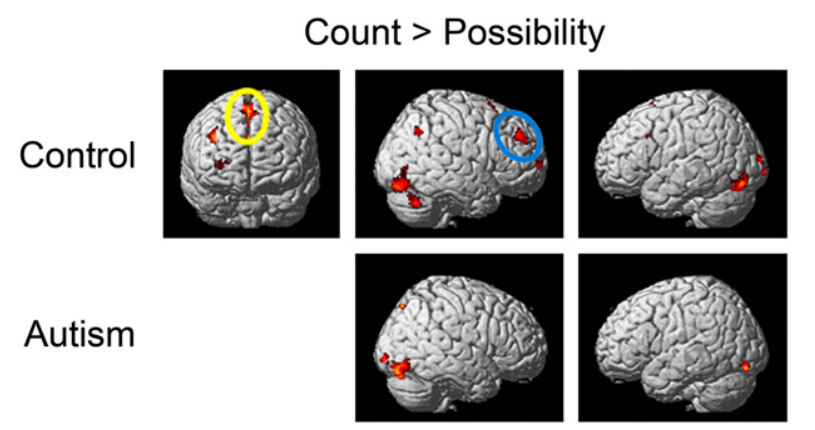

(b) Overall Similarity of Activation in the Two Tasks

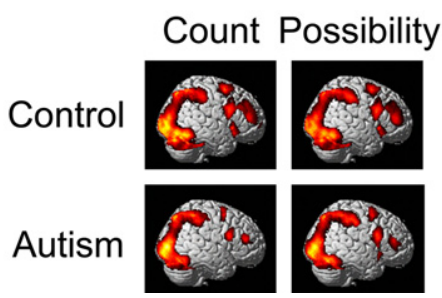

Fig. 2. (a) Differences of activation between the two tasks (Count $>$ Possibility) for control (top row) and autism group (bottom row). The yellow ellipse indicates greater activation in the medial frontal area and the blue ellipse indicates great activation in the right superior frontal region. (b) Overall similarity of activation in the two tasks. The activation was bilateral, but only the right hemisphere was shown here to illustrate the overall similarity. The top row presents the activation maps of count (left) and possibility (right) tasks for control participants; the bottom row shows the activation maps of count (left) and possibility (right) tasks for the participants with autism. $p<0.001$.

\subsection{Distribution of activation}

\subsubsection{Group differences in between-task contrasts}

Both groups have more activation in the count task than in the possibility task in the bilateral occipital and fusiform areas and right posterior parietal area, and have more activation in the possibility task than in the count task in the bilateral postcentral, bilateral frontal, and left fusiform brain regions. However, only for the control participants, there was greater activation in the medial frontal (yellow ellipse) and right superior frontal (blue ellipse) in the count task than in the possibility task (Fig. 2a). Analyses of the percent signal change (PSC) in the medial frontal ROI revealed an interaction between group and task $(F(1,28)=4.47$, $p<0.05)$. Control participants had a reliably greater mean PSC in the medial frontal ROI in the count task (mean $=0.27$ ) than in the possibility task (mean $=0.20 ; t(14)=3.47, p<0.01)$; but there was no such difference in the autism group (count: mean $=0.23$; possibility: mean $=0.22 ; t(14)=0.41, p>0.10$ ). Furthermore, for the control group, the PSC difference between count and possibility tasks in the medial frontal ROI was positively correlated with participants' Vandenberg scores $(r=0.62, p<0.05$ ) (as shown in Fig. 3 ); for the autism group, the correlation had a negative trend $(r=-0.45, p<0.10)$. The difference in correlations between the two groups was significant $(p<0.05)$.

\subsubsection{Count vs. possibility task}

Despite the between-task differences in activation described above, control participants recruited strikingly similar frontal, parietal and occipital regions in the two tasks (Fig. 2b). This similarity confirmed that the global (3-D) processing occurred not only in the possibility but also in the count task. Participants with autism also

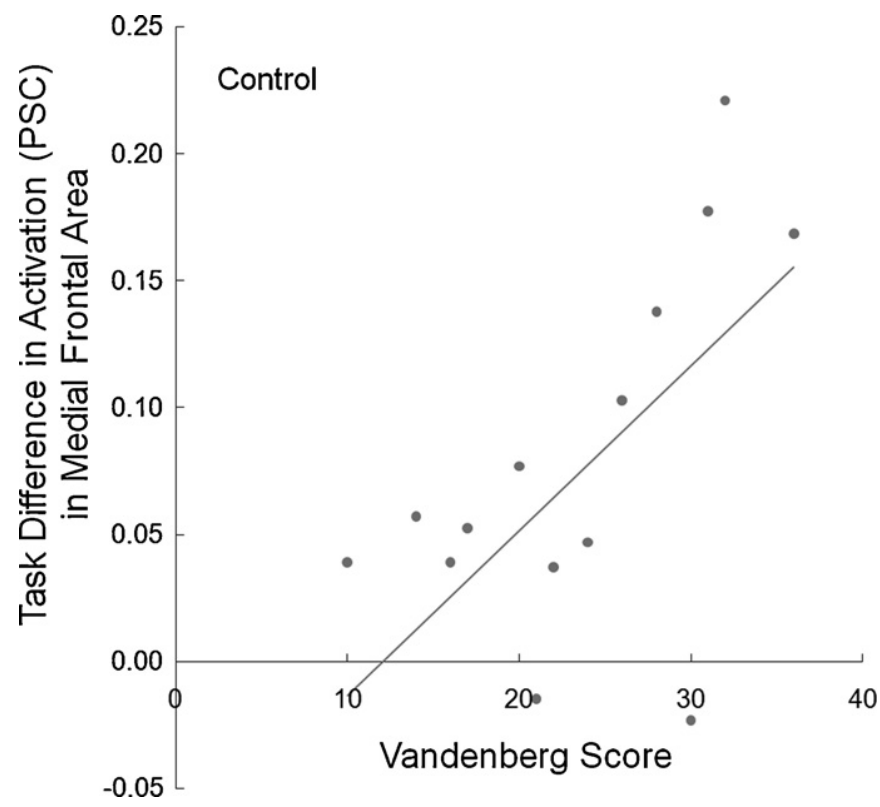

Fig. 3. Positive correlation between Vandenberg score and the activation (PSC) difference between count and possibility tasks in the medial frontal ROI in controls. $r=0.62, p<0.05$.

recruited similar areas in the two tasks (Fig. 2b). In the count task, participants with autism showed less activation than controls in the right fusiform gyrus and left inferior parietal regions; in the possibility task, participants with autism showed less activation than controls in the right fusiform region. There was no brain region in which participants with autism showed more activation than controls in either task.

\subsection{Functional connectivity}

Based on the group differences in the between-task contrasts described above, the functional ROIs were grouped into 4 sets: medial frontal ROI, ROIs in other frontal region (bilateral dorsolateral prefrontal, insula, inferior frontal, middle frontal), postcentral ROIs, and ROIs in other posterior regions (bilateral fusiform, inferior parietal, precuneus, inferior occipital, and middle occipital). Functional connectivities between the medial frontal ROI and ROIs in other brain regions, as well as between the postcentral ROIs and ROIs in other brain regions were computed. The main synchronization finding was that the count task elicited higher functional connectivities than the possibility task in the control group but not in the autism group.

Unlike the autism group, control participants had higher functional connectivity between the medial frontal ROI and posterior (visuospatial) ROIs in the count task than in the possibility task $(p<0.001$, See Fig. 4 ; there was no such difference in autism, $p>0.10)$. There was a reliable Group $\times$ Task interaction in these functional connectivities $(F(1,28)=4.63, p<0.05) .{ }^{1}$ Similarly, for the functional connectivities between the medial frontal ROI and the set of other frontal ROIs, control participants had higher functional connectivities in the count task than in the possibility task $(p<0.001)$ and there was no such difference in autism $(p>0.10)$. However, the Group $\times$ Task interaction was not significant $(F(1$, $28)=1.98, p>0.10)$.

\footnotetext{
1 To demonstrate the robustness of this finding over alternative methods of defining ROIs, this analysis was repeated using anatomically defined ROIs, namely Anatomical Automatic Labeling (AAL) masks (Tzourio-Mazoyer et al., 2002). The Group $\times$ Task interaction was again significant $(F(1,28)=7.69, p<0.05)$.
} 


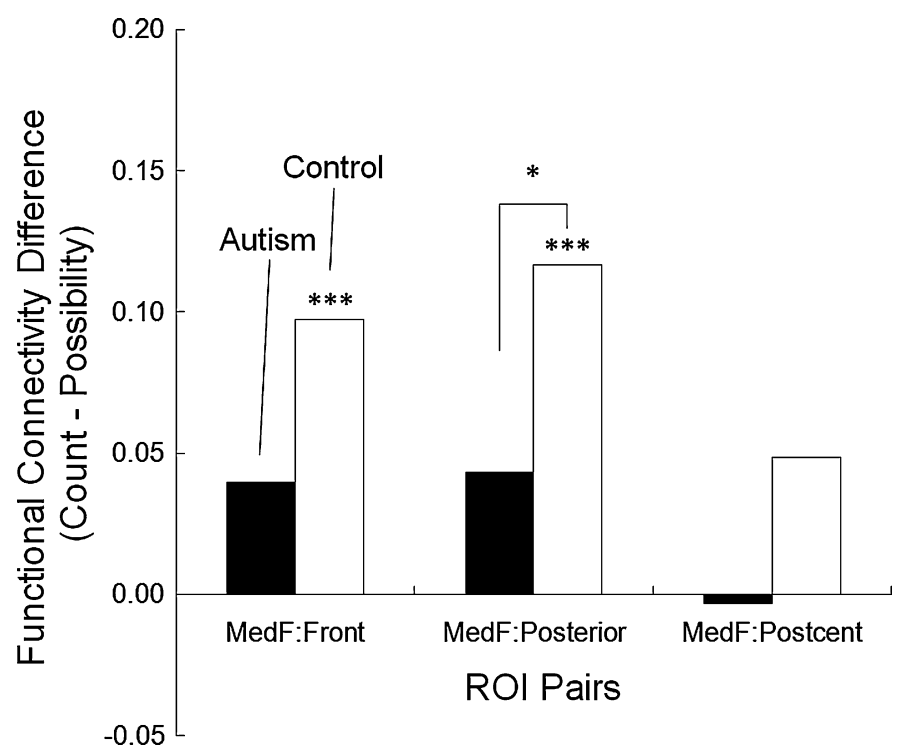

Fig. 4. Functional connectivity differences between the count and possibility tasks between the medial frontal ROI and ROIs in the other brain regions. Both withingroup and between-group differences are shown. ${ }^{* * *} p<0.001,{ }^{*} p<0.05$, blank, ns.

Both groups had higher functional connectivities in the possibility task than in the count task between the postcentral and frontal ROIs ( $p<0.05$ for both groups), as well as between the postcentral and posterior ROIs ( $p<0.05$ for both groups). There was no Group $\times$ Task interaction $(p>0.10)$ for the functional connectivities between the postcentral and frontal/posterior ROIs.

Following the theory of frontal-posterior underconnectivity in autism (Just, Cherkassky, Keller, \& Minshew, 2004; Just, Cherkassky, Keller, Kana, \& Minshew, 2007), we tested for a group difference in frontal-posterior functional connectivity. The functional ROIs were grouped into a set of frontal ROIs (medial frontal, bilateral dorsolateral prefrontal, insula, inferior frontal, and middle frontal) and posterior ROIs (bilateral fusiform, inferior parietal, postcentral, precuneus, inferior occipital, and middle occipital). There was no group difference in the frontal-posterior functional connectivity in either task (possibility task: autism group, mean $=0.82$; control group, mean $=0.84, t(13)=0.40, p>0.10$; count task: autism group, mean $=0.81$; control group, mean $=0.84, t(13)=0.48, p>0.10)$. Thus, unlike the findings in many studies of autism using higherlevel cognitive tasks involving language and executive function (Just et al., 2004, 2007; Kana, Keller, Cherkassky, Minshew, \& Just, 2006; Kana, Keller, Minshew, \& Just, 2007; Kana, Keller, Cherkassky, Minshew, \& Just, 2009; Mason, Williams, Kana, Minshew, \& Just, 2008), the current pair of tasks showed very slight and not significant frontal-posterior underconnectivity in autism, perhaps because of lesser reliance on frontal areas in these perceptual tasks.

\subsection{Behavioral results}

The two groups were comparable in their behavioral performance. There was no group difference in either reaction times $(F<1)$ or error rates $(F(1,28)=2.40, p>0.10)$. In both groups, responses were slower in the count task (autism group: mean $=3858 \mathrm{~ms}$, se $=229$; control group: mean $=3981 \mathrm{~ms}$, se $=265$; $F(1,28)=12.21, p<0.01$ ) than in the possibility task (autism group: mean = $3432 \mathrm{~ms}$, se =239; control group: mean = $3373 \mathrm{~ms}$, se = 171). Although the slowing effect in the count task appeared to be larger in controls than in autism, the interaction between group and task was not statistically significant $(F<1)$. The participants with autism made fewer errors in the count task (mean $=8.0 \%$, se $=1.9 \%$ ) than in the possibility task (mean $=9.7 \%$, se $=1.8 \%$ ), and the controls made

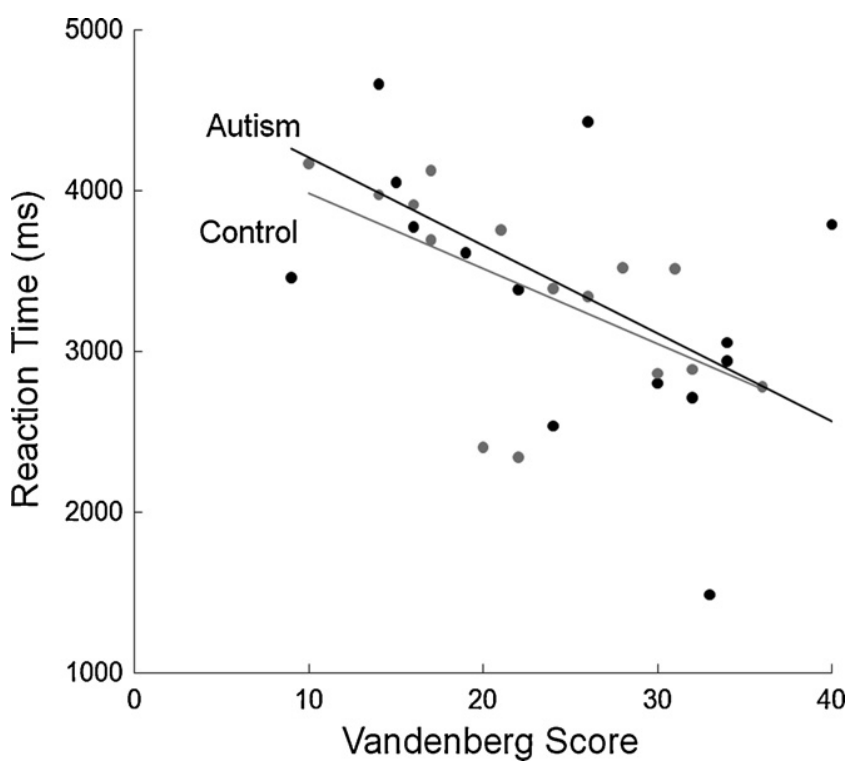

Fig. 5. Negative correlation between reaction times and Vandenberg score on the possibility task in autism group (black) and control group (grey). Autism: $r=-0.59$, $p<0.05$; control group: $r=-0.59, p<0.05$.

more errors in the count task (mean $=6.7 \%$, se $=1.3 \%$ ) than in the possibility task (mean $=5.3 \%$, se $=1.5 \%$ ), consistent with the more sensitive neuroimaging findings described above. However, there was no main effect of task $(F<1)$ or interaction between group and $\operatorname{task}(F(1,28)=1.19, p>0.10)$.

There was no group difference in the Vandenberg scores $(t$ $(28)=0.49, p>0.10$ ). The reaction times in the possibility task were negatively correlated with Vandenberg scores in both the autism $(r=-0.59, p<0.05)$ and control group $(r=-0.59, p<0.05)$ (see Fig. 5). That is, participants with higher Vandenberg scores made faster judgments concerning the possibility of figures. No such correlation was found in the count task for either group.

\section{Discussion}

The current study investigated the neural basis of the atypicality of perceptual processing in autism, examining the impact of 3-D structural information on the activation in a lower-level line-counting task. In the count task (compared to the possibility task), the participants with autism did not show increased activation in the superior frontal and medial frontal brain regions, nor increased functional connectivity between the medial frontal and posterior regions, unlike the control group, suggesting systematic group differences in the neural basis of the count task. We suggest that the group difference occurred in the count task because the autism group (unlike the control group) was less subject to interference from global processing. By contrast, in the possibility task that explicitly focused on the 3-D processing, the participants with autism were similar to the matched controls with respect to behavioral performance, brain activation patterns, and functional connectivity.

\subsection{Count vs. possibility task}

The two tasks in this experiment required attention to be focused on different types of properties of the stimuli. The count task required attending only to individual lines of different colors (feature-level rather than global-oriented processing) and deciding which color had more lines. By contrast, the possibility task required a more global assessment of the stimulus configuration, 
requiring one to attend to the global properties of an object. Both groups performed the tasks by recruiting similar networks of brain areas: more posterior regions (occipital, inferior temporal, and posterior parietal) in the count task, suggesting that detailed visual analysis was performed to process the local features (i.e., color of the lines), and more frontal, parietal, and temporal brain regions in the possibility task, suggesting the involvement of the extensive brain network for the global spatial processing. At the same time, for both groups, there was a substantial similarity between the activation patterns for the two tasks, reflecting the presence of the 3-D processing in both cases, despite it being only a distraction in the line-counting task, particularly so for the control participants.

\subsection{Reduced global to local interference in autism}

As noted previously in the count task, the 3-D configurations are task-irrelevant and play a similar role to the embedding figure in the embedded figure task, or the distractors in a visual search task. In the control group, the automatic processing of the 3-D structure of the figure presumably interfered with the task goal of searching for and comparing colored contours; thus the high-level executive brain regions were recruited and functioning. The medial frontal gyrus is generally associated with high-level executive functions and decision making (Brass, Wenke, Spengler, \& Waszak, 2009; Ridderinkhof, Ullsperger, Crone, \& Nieuwenhuis, 2004; Rushworth, Kennerley, \& Walton, 2005; Stuss \& Alexander, 2007). The greater activation in the superior frontal and medial frontal brain region in the count-possibility contrast may reflect the role of these regions in the modulation of attention and/or resolution of conflict induced by the 3-D computation in control. The positive correlation in the control group between medial frontal activation (percent signal change in the medial frontal brain regions in the count-possibility contrast) and the Vandenberg scores supports the cognitive control role of this region. That is, the control participants with higher spatial ability (indicated by higher Vandenberg scores) may have done more suppression of the global 3-D processing and directed their attention more to the searching for and counting color contours. Consistent with this account, there was an increased synchronization between the medial frontal and posterior visuospatial regions in the control group. In addition, the greater activation in the superior medial frontal brain region may also reflect working memory resources used for searching and comparing the numbers of red vs. green lines. The superior frontal gyrus has been found to be involved in tasks of working memory (Petrides, 2005), notably spatial working memory (Courtney, Petit, Maisog, Ungerleider, \& Haxby, 1998; Haxby, Petit, Ungerleider, \& Courtney, 2000).

By contrast, in the autism group, the 3-D stimulus configuration in the count task may have had a diminished effect on the task goal of search and comparison, due to a lack or lower level of automaticity in figural processing, resulting in a relative autonomy of local processing in autism. This is evidenced by a lower level of activation of executive brain regions and synchronization between executive and posterior visuospatial regions in autism in the countpossibility contrast. Unlike control participants, who may have had to suppress the background information and focus their attention on searching and comparing colored lines in the count task, participants with autism may not have needed to do any suppression. The trend of a negative correlation between Vandenberg scores and percent signal change in the medial frontal brain regions in the count-possibility contrast in autism suggest that individuals with autism who have lower spatial ability may depend on increased use of cognitive attentional resources for searching and comparing colored contours, resulting in greater activation in the medial frontal region. By contrast, individuals with autism who have higher spatial ability may perform the count task in a more visuospatial manner.
The original weak central coherence (WCC) theory (Frith, 1989; Frith \& Happé, 1994) would argue that the diminished influence from the task-irrelevant global information in autism is due to a deficit in the central coherence of the information processing system. However, the autism group had similar behavioral performance and brain activation patterns as the control group in the possibility task, indicating that the autism group was able to perform the higher-level global task as well as the control group. The diminished influence from the task-irrelevant 3-D information in the count task in autism was not due to the weak coherence of the central information processing system. The EPF account (Mottron et al., 2006) and some version of the current WCC theory (Happé \& Frith, 2006) would argue that a local bias in cognitive processing style (WCC theory) or perception (EPF account) kept the individuals with autism from experiencing interference from global information and allowed them to attend to the local details of the visual stimuli (color of lines) to fulfill the count task. Our imaging results fit in well with the framework of such accounts of hierarchical processing.

\section{Conclusion}

The participants with autism performed the line-counting task in a local visual-spatial manner and were less or not at all affected by the global processing of the 3-D figure. In comparison, the control participants had to suppress the automatic processing of irrelevant global information and increased their attention on finding and counting colored lines. While accumulating behavioral evidence has demonstrated that individuals with autism are less hindered by interference from task-irrelevant global information, this is the first neuroimaging study that provides direct evidence of brain manifestations of reduced global-to-local perceptual processing in autism.

\section{Acknowledgements}

This research was supported by the Autism Centers of Excellence Grant (HD055748) from the National Institute of Child Health and Human Development. We appreciate the assistance of current and former members of the Center for help in conducting the experiments. In particular, we would like to thank Justin Abernethy for his assistance in preparing materials, Jennifer Moore for editorial comments, Diane Williams for extensive discussions, and Tim Keller for his excellent technical support and comments on earlier versions of the manuscript. We would also like to express our sincere appreciation for the time and effort of the participants and their families in making this research possible.

\section{References}

American Psychiatric Association. (1994). Diagnostic and statistical manual of mental disorders (4th ed. (DSM-IV)). Washington, DC: APA.

Behrmann, M., Avidan, G., Leonard, G. L., Kimchi, R., Luna, B., Humphreys, K., et al (2006). Configural processing in autism and its relationship to face processing. Neuropsychologia, 44, 110-129.

Brass, M., Wenke, D., Spengler, S., \& Waszak, F. (2009). Neural correlates of overcoming interference from instructed and implemented stimulus-response associations. The Journal of Neuroscience, 29, 1766-1772.

Brosnan, M. J., Scott, F. J., Fox, S., \& Pye, J. (2004). Gestalt processing in autism: Failure to process perceptual relationships and the implications for contextual understanding. Journal of Child Psychology and Psychiatry, 4, 54-59.

Courtney, S. M., Petit, L., Maisog, J. M., Ungerleider, L. G., \& Haxby, J. V. (1998). An area specialized for spatial working memory in human frontal cortex. Science, 279, 1347-1351.

Damarla, S. R., Keller, T. A., Kana, R. K., Cherkassky, V. L., Williams, D. L., \& Minshew, N. J. (2010). Cortical underconnectivity coupled with preserved visuospatial cognition in autism: Evidence from an fMRI study of an embedded figures task. Autism Research, 3, 273-279. 
Friston, K., Ashburner, J., Frith, C., Poline, J.-B., Heather, J., \& Frackowiak, R. (1995). Spatial registration and normalization of images. Human Brain Mapping, 2, 165-189.

Frith, U. (1989). Autism: Explaining the enigma. Oxford, UK: Blackwell.

Frith, U., \& Happé, F. (1994). Autism: Beyond "theory of mind". Cognition, 50, 115-132.

Happé, F. G., \& Booth, R. D. (2008). The power of the positive: Revisiting weak coherence in autism spectrum disorders. The Quarterly Journal of Experimental Psychology, 61, 50-63.

Happé, F. G., \& Frith, U. (2006). The weak coherence account: Detailed-focused cognitive style in autism spectrum disorders. Journal of Autism and Developmental Disorders, 36, 5-25.

Haxby, J. V., Petit, L., Ungerleider, L. G., \& Courtney, S. M. (2000). Distinguishing the functional roles of multiple regions in distributed neural systems for visual working memory. Neuroimage, 11, 380-391.

Hubl, D., Bolte, S., Feineis-Matthews, S., Lanfermann, H., Federspiel, A., Strik, W., et al. (2003). Functional imbalance of visual pathways indicates alternative face processing strategies in autism. Neurology, 61, 1232-1237.

Jolliffe, T., \& Baron-Cohen, S. (1997). Are people with autism and Asperger syndrome faster than normal on the embedded figures test? Journal of Child Psychology $\mathcal{E}^{\prime}$ Psychiatry, 38, 527-534.

Joseph, R. M., \& Tanaka, J. (2003). Holistic and part-based face recognition in children with autism. Journal of Child Psychology and Psychiatry, 44, 529-542.

Just, M. A., Cherkassky, V. L., Keller, T. A., \& Minshew, N. J. (2004). Cortical activation and synchronization during sentence comprehension in high-functioning autism: Evidence of underconnectivity. Brain, 127, 1811-1821.

Just, M. A., Cherkassky, V. L., Keller, T. A., Kana, R. K., \& Minshew, N. J. (2007). Functional and anatomical cortical underconnectivity in autism: Evidence from an fMRI study of an executive function task and corpus callosum morphometry. Cerebral Cortex, 17, 951-961.

Kana, R. K., Keller, T. A., Cherkassky, V. L., Minshew, N. J., \& Just, M. A. (2006). Sentence comprehension in autism: Thinking in pictures with decreased functional connectivity. Brain, 129, 2484-2493.

Kana, R. K., Keller, T. A., Minshew, N. J., \& Just, M. A. (2007). Inhibitory control in high functioning autism: Decreased activation and underconnectivity in inhibition networks. Biological Psychiatry, 62, 198-206.

Kana, R. K., Keller, T. A., Cherkassky, V. L., Minshew, N. J., \& Just, M. A. (2009). Atypical frontal-posterior synchronization of theory of mind regions in autism during mental state attribution. Social Neuroscience, 4, 135-152.

Lee, P. S., Foss-Feig, J., Henderson, J. G., Kenworthy, L. E., Gilotty, L., Gaillard, W. D., et al. (2007). Atypical neural substrates of embedded figures task performance in children with autism spectrum disorder. NeuroImage, 38, 184-193.

Lord, C., Rutter, M., \& Le Couteur, A. (1994). Autism diagnostic interview-revised: A revised version of a diagnostic interview for caregivers of individuals with possible pervasive developmental disorders. Journal of Autism and Developmental Disorders, 24, 659-685.

Lord, C., Risi, S., Lambrecht, L., Cook, E. H., Leventhal, B. L., DiLavore, P. C., et al. (2000). The autism diagnostic observation schedule-generic: A standard measure of social and communication deficits associated with the spectrum of autism. Journal of Autism and Developmental Disorders, 30, 205-223.

Manjaly, Z. M., Marshall, J. C., Stephan, K. E., Gurd, J. M., Zilles, K., \& Fink, G. R. (2003). In search of the hidden: An fMRI study with implications for the study of patients with autism and with acquired brain injury. NeuroImage, 19, 674-683.

Manjaly, Z. M., Bruning, N., Neufang, S., Stephan, K. E., Brieber, S., Marshall, J. C., et al. (2007). Neurophysiological correlates of relatively enhanced local visual search in autistic adolescents. NeuroImage, 35, 283-291.
Mason, R. A., Williams, D. L., Kana, R. K., Minshew, N. J., \& Just, M. A. (2008). Theory of mind disruption and recruitment of the right hemisphere during narrative comprehension in autism. Neuropsychologia, 46, 269-280.

Minshew, N. J. (1996). Autism. In B. O. Berg (Ed.), Principles of child neurology (pp. 1713-1729). New York, USA: McGraw-Hill.

Mottron, L., Belleville, S., \& Ménard, E. (1999). Local bias in autistic subjects as evidenced by graphic tasks: Perceptual hierarchization or working memory deficit? Journal of Child Psychology and Psychiatry, 40, 743-755.

Mottron, L., Dawson, M., Soulières, I., Hubert, B., \& Burack, J. A. (2006). Enhanced perceptual functioning in autism: An update, and eight principle of autistic perception. Journal of Autism and Developmental Disorders, 36, 27-43.

O’Riordan, M. A., Plaisted, K. C., Driver, J., \& Baron-Cohen, S. (2001). Superior visual search in autism. Journal of Experimental Psychology: Human Perception and Performance, 27, 719-730.

Petrides, M. (2005). Lateral prefrontal cortex: Architectonic and functional organization. Philosophical Transactions of the Royal Society, 360, 781-795.

Plaisted, K. C. (2000). Aspects of autism that theory of mind cannot easily explain. In S. Baron-Cohen, H. Tager-Flusberg, \& D. J. Cohen (Eds.), Understanding other minds: Perspectives from autism and cognitive neuroscience. Oxford: Oxford University Press.

Plaisted, K. C. (2001). Reduced generalization in autism: An alternative to weak central coherence. In J. A. Burack, T. Charman, N. Yirmiya, \& P. R. Zelazo (Eds.), The development of autism: Perspectives from theory and research (2nd ed., pp. 149-169). New Jersey: Lawrence Erlbaum.

Plaisted, K., O'Riordan, M., \& Baron-Cohen, S. (1998). Enhanced visual search for a conjunctive target in autism: A research note. Journal of Child Psychology and Psychiatry, 39, 777-783.

Reitan, R. M. (1985). In A. Z. Tucson (Ed.), Halstead-Reitan neuropsychological test battery. Tucson, AZ: Reitan Neuropsychological Laboratories, University of Arizona.

Ridderinkhof, K. R., Ullsperger, M., Crone, E. A., \& Nieuwenhuis, S. (2004). The role of the medial frontal cortex in cognitive control. Science, 306, 443-447.

Rinehart, N. J., Bradshaw, J. L., Moss, S. A., Brereton, A. V., \& Tonge, B. J. (2000). Atypical interference of local detail on global processing in high-functioning autism and Asperger's disorder. Journal of Child Psychology \& Psychiatry, 41, 769-778.

Ring, H., Baron-Cohen, S., Williams, S., Wheelwright, S., Bullmore, E., Brammer, M., et al. (1999). Cerebral correlates of preserved cognitive skills in autism. A functional MRI study of embedded figures task performance. Brain, 122, 1305-1315.

Rushworth, M. F., Kennerley, S. W., \& Walton, M. E. (2005). Cognitive neuroscience: Resolving conflict in and over the medial frontal cortex. Current Biology, R54-R56.

Shah, A., \& Frith, U. (1993). Why do autistic individuals show superior performance on the block design task? Journal of Child Psychology and Psychiatry, 34, 1351-1364.

Shepard, R. N., \& Metzler, J. (1971). Mental rotation of three-dimensional objects. Science, 19, 701-703.

Stuss, D., \& Alexander, M. (2007). Is there a dysexecutive syndrome? Philosophical Transactions of the Royal Society, 362, 901-915.

Tzourio-Mazoyer, N., Landeau, B., Papathanassiou, D., Crivello, F., Etard, O., Delcroix, N., et al. (2002). Automated anatomical labeling of activations in SPM using a macroscopic anatomical parcellation of the MNI MRI single-subject brain. Neuroimage, $15,273-289$.

Vandenberg, S. G., \& Kuse, A. R. (1978). Mental rotations, a group test of threedimensional spatial visualization. Perceptual \& Motor Skills, 47, 599-604. 